\title{
Seven year follow up of children presenting to the accident and emergency department with irritable hip
}

\author{
Anthony Mattick, Alastair Turner, James Ferguson, Thomas Beattie, Jacqueline Sharp
}

Department of

Accident and

Emergency Medicine,

Aberdeen Royal

Infirmary, Aberdeen

A Mattick

A Turner

J Ferguson

J Sharp

\section{Department of}

Accident and

Emergency Medicine, Royal Hospital for Sick Children, Edinburgh

T Beattie

Correspondence to:

Mr Anthony Mattick,

Specialist Registrar,

Department of Accident and

Emergency Medicine, Royal

Infirmary of Edinburgh, 1

Lauriston Place, Edinburgh

EH3 9YW.

Accepted 20 March 1999

\begin{abstract}
Objective-To assess an established protocol for managing children with irritable hip in the accident and emergency department.

Methods-Retrospective seven year follow up of all children managed under an established hip pain protocol. The main outcome measure was of failure of the protocol to identify serious pathology.

Results-A total of 103 children met the criteria for assessment using the protocol. Sixty were allowed home, and outpatient follow up arranged. All of these children except one were diagnosed as having transient synovitis. This child had Perthes' disease and was diagnosed at first presentation. Forty three children were admitted, with eight subsequently having a diagnosis other than transient synovitis of the hip. It was possible to review 80 children seven years later. Of these children no long term problems were encountered.

Conclusion-The protocol used in the department for children with irritable hip is successful in identifying those children with transient synovitis of the hip, or other benign causes, and therefore not requiring hospital admission. Long term follow up showed that no serious pathology was missed.

(F Accid Emerg Med 1999;16:345-347)
\end{abstract}

Keywords: irritable hip; transient synovitis

serious disorders associated with the limping child (table 1). ${ }^{25}$

Because of the serious nature of the many potential diagnoses it has been suggested that those children all be admitted. ${ }^{26}$ Over recent years, however, practice has tended to a less aggressive approach, enabling many children with transient synovitis to be managed as outpatients using analgesia and rest. This we feel is a more appropriate approach to managing the condition and is the preferred choice of most children and parents. The problem therefore is to produce a protocol, which will effectively and accurately screen for transient synovitis and other benign conditions, yet identify significant pathology. Our management protocol (fig 1) reflects this attempt. This paper presents an audit of our experience applying this protocol and is a seven year follow up study to establish its effectiveness.

\section{Methods}

All children presenting with hip pain or suspected hip pathology, over an 11 month period during 1989 to the Royal Aberdeen Children's Hospital, were identified as part of a previously unpublished internal audit. All were managed according to the protocol in fig 1 . After seven years all records were retrieved and patients followed up by contacting general practitioners (GPs), phone contact, and A\&E inpatient chart review. The population base studied is known to be stable and previous long term studies have commented on this. ${ }^{7}$ The main outcome measure was the failure of the protocol to detect serious pathology either in the short term or subsequently.

The limping child with no known history of trauma is a common presentation or referral to the accident and emergency (A\&E) department, with most having hip pathology. ${ }^{1}$ Transient synovitis of the hip is by far the commonest cause. ${ }^{23}$ First described by Lovett and Morse in $1892,{ }^{4}$ it is a benign self limiting disease with no long term complications in the majority of cases. However, the diagnosis should be made only after exclusion of more

Table 1 Causes of atraumatic limp in childhood

Transient synovitis of the hip

Septic arthritis

Perthes' disease

Slipped upper femoral epiphysis

Osteomyelitis

Juvenile rheumatoid arthritis

Malignancy, including leukaemia, primary or secondary bone tumours

Sickle crisis

Referred pain, for example, testicular torsion, appendicitis

\section{Results}

A total of 103 children attended the $A \& E$ department with hip pain or suspected hip pathology between 1 January 1989 and 30 November 1989 . This represented $0.64 \%$ of all those attending. Follow up was achieved in 80 cases. The uncontactable children (23 in total) were compared with these for age, sex, and diagnosis using the Mann-Whitney test for non-parametric, unpaired data. There was no significant difference between the two groups, indicating that there was no bias in selection of the contactable group.

Of the original total of $103,77(75 \%)$ were male and $26(25 \%)$ were female, giving a ratio of 3:1. The age of the patients ranged from 11 months to 15 years with a median age of 5 years. The left hip was affected in $53 \%$ of cases, the right hip in $45 \%$, and both hips in $2 \%$. The 
median duration of stay for those children admitted with a subsequent diagnosis of transient synovitis was 3 days (range 1-8 days). Simplistic extrapolation of this figure to the 59 children with transient synovitis treated as outpatients shows 177 days of hospital admission were saved using the protocol.

\section{ANALYSIS OF INITIAL DIAGNOSIS}

Children allowed home for outpatient follow up

A total of 60 patients (58\%) were allowed home on the day of presentation. Fifty nine had normal parameters within the framework of the protocol, and the diagnosis of presumed transient synovitis given. Review, according to protocol, occurred subsequently. One further patient with early radiological signs of Perthes' disease was allowed home after admission was refused by the parents. Prompt orthopaedic outpatient follow up was arranged for her.

\section{Children admitted}

Forty three patients $(42 \%)$ met the criteria for admission. Specific criteria for those admitted are shown in table 2 and their subsequent diagnoses in table 3.

LONG TERM FOLLOW UP

Of the original total of 103 children it was possible to contact 80 for follow up questioning seven years later. Of the 80 children, $33 \mathrm{had}$

Hip protocol

\begin{tabular}{|lcccc|}
\hline \multicolumn{1}{l}{} & & & \\
\hline $\begin{array}{l}\text { Range of movement } \\
<75 \% \text { of normal hip }\end{array}$ & $\begin{array}{c}\text { Temperature } \\
>37.5^{\circ} \mathrm{C}\end{array}$ & $x$ Ray & WCC & ESR \\
\hline
\end{tabular}

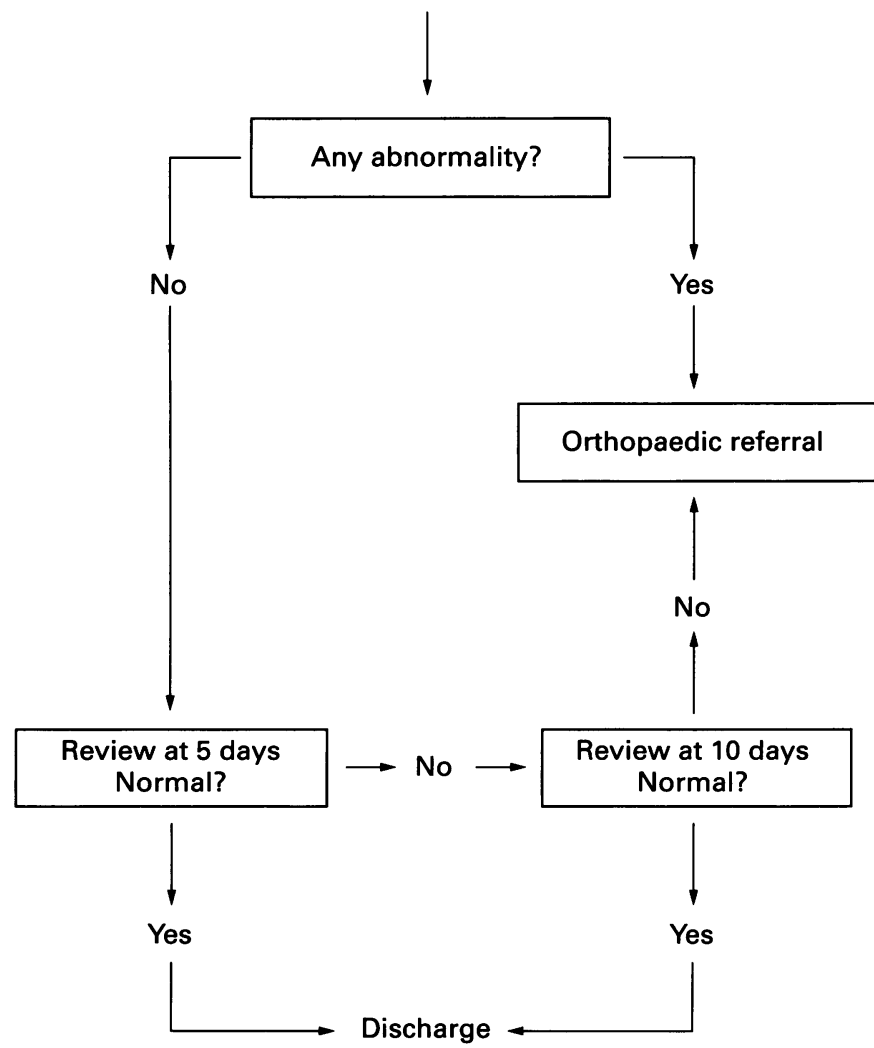

Figure 1 Management protocol $(E S R=$ erythrocyte sedimentation rate; $W C C=$ white cell count).
Table 2 Specific criteria that lead to admission under the protocol

\begin{tabular}{lc}
\hline Parameter & No of children \\
\hline Severe limitation of movement & 27 \\
ESR $>20 \mathrm{~mm} /$ hour & 4 \\
WCC $>13.0 \times 10^{9} / 1$ & 5 \\
Temperature $>37.5^{\circ} \mathrm{C}$ & 4 \\
Abnormal radiography & 1 \\
Combination & 2
\end{tabular}

ESR = erythrocyte sedimentation rate; $\mathrm{WCC}=$ white cell count.

Table 3 Subsequent diagnoses of those children who were admitted

\begin{tabular}{ll}
\hline Disease & No of children \\
\hline Perthes' disease & 3 \\
Septic arthritis & 2 \\
Trochanteric bursitis & 1 \\
Kawasaki's disease & 1 \\
Glandular fever & 1 \\
Transient synovitis & 35 \\
Total & 43 \\
\hline
\end{tabular}

been admitted under the protocol $(77 \%$ of the total admitted), and 47 had been allowed home ( $78 \%$ of the total allowed home).

\section{Admitted cohort ( 33 children)}

The group that was followed up included all those who had been admitted with a diagnosis other than transient synovitis, except for two children with Perthes' disease who were lost to follow up.

Out of the 27 in this group originally admitted with an eventual diagnosis of transient synovitis, four went on to develop further recurrence; three attended $A \& E$ and were discharged using the protocol, while one did not seek medical help. None of the 27 developed any long term complications.

None of those admitted with a diagnosis other than transient synovitis went on to develop further problems after initial treatment.

\section{Allowed home for outpatient follow up cohort} (47 children)

Forty seven children were followed up not having been admitted. This group included the child with Perthes' disease whose parents had refused admission. All in this group apart from the child with Perthes' disease were diagnosed as having transient synovitis; 10 had since had a similar recurrence of whom three reattended $\mathrm{A} \& \mathrm{E}$ and were again allowed home via the protocol. Four attended their GP and were advised to rest at home, and three did not seek medical help. None of the 46 developed any long term problems and were fully well at seven years.

The patient with Perthes' disease did not require any medical intervention other than six monthly radiographs and no long term problems were encountered.

\section{Discussion}

By using a protocol based on discharging relatively asymptomatic children and admitting those with one or more danger signs we hoped to identify patients appropriately. 
The exact cut off levels of erythrocyte sedimentation rates and white cell counts for referral were those above which the local haematology laboratory considered abnormal. Other studies have broadly substantiated the values used..$^{8-10}$ It is departmental policy to consider those with an axillary temperature above $37.5^{\circ} \mathrm{C}$ as being pyrexial, a figure comparable with other studies. ${ }^{11}$ Other parameters such as limitation of joint movement and significant pain are also useful due to their association with infection.

Due to the stable population base in this region it was possible to achieve a follow up rate of almost $80 \%$ at seven years. When comparing the 23 children lost to follow up with those 80 who were followed up, it was found that they were comparable with regard to age, sex, and diagnosis.

Using the protocol described, $58 \%$ of children were allowed home from the A\&E department and treated as outpatients, without significant pathology being missed or adverse long term affects. All those children found to have significant pathology were detected and admitted under the protocol, with the exception of one girl with Perthes' disease whose parents refused. Reassuringly, serious pathology was found only in seven patients: four with Perthes' disease, two with septic arthritis, and one with Kawasaki's disease. No long term problems were found with these children or with the others who were admitted. However two of the patients with Perthes' disease were unfortunately among those lost to follow up.

The protocol was developed before studies demonstrated that the use of ultrasound, ${ }^{12}{ }^{13}$ and ultrasound guided aspiration of joint effusions, may be useful in both the diagnosis and symptomatic relief of the limping child. Ultrasonography is by far superior at detecting joint effusions compared with conventional radiography, ${ }^{14}$ but relies on the availability of an experienced ultrasonographer. Any protocol used now would have to consider ultrasound as an integral part.

\section{Conclusion}

No one clinical finding or investigation can be used in managing the limping child who attends the A\&E department. The protocol was found to be an effective method of preventing unnecessary admissions of children who presented to the $A \& E$ department with a limp. More importantly no serious pathology was missed. Ultrasonography as a diagnostic tool was not a viable option when the protocol was established but currently would be our first investigation of choice.

Conflict of interest: none.

Funding: none.

1 Fischer SU, Beattie TF. Epidemiology of childhood limp presenting to a paediatric A\&E department. $\mathcal{F}$ Bone foint

Surg $\mathrm{Br}$ (in press).
2 Briggs RD, Baird KS, Gibson PH. Transient synovitis of the hip joint. $\mathcal{F} R$ Coll Surg Edinb 1990;35:48-50.

3 Landin L, Danielsson L, Wattsgard C. Transient synovitis of the hip, its incidence, epidemiology and relation to Perthes' disease. F Bone foint Surg Br 1987;69:238-41.

4 Lovett RW, Morse JL. A transient or ephemeral form of hip disease with a report of cases. Boston Medical and Surgical fournal 1892;127:161.

5 Bickerstaff D. Irritable hip. Surgery 1993;11:485-8.

6 Hollingworth P. Differental diagnosis and management of hip pain in childhood. Br f Rheumatol 1995;34:78-82.

7 Ross S, Godden D, McMurray D, et al. Social effects of wheeze in childhood: a 25 year follow up. $B M \mathcal{F} 1992 ; 305$ : 545-8.

8 Del Beccaro M, Champoux A, Bockers T, et al. Septic arthritis versus transient synovitis of the hip: the value of
and arthritis versus transient synovitis of the hip: the value of 22.

9 Taylor GR, Clarke MMP. Management of irritable hip: a review of hospital admission policy. Arch Dis Child 1994;71:59-63.

10 Kunnamo I, Kallio P, Pelkonen P, et al. Clinical signs and laboratory tests in the differential diagnosis of arthritis in children. Am $\mathcal{F}$ Dis Child 1987;141:34-40.

11 Whybrew K, Murray M, Morley C. Diagnosing fever by touch: observational study. BMF 1998;317:321.

12 Zawin JK, Hoffer F, Rand F, et al. Joint effusion in children with an irritable hip: US diagnosis and aspiration. Radiology 1993;187:459-63

13 Fink AM, Berman L, Edwards D, et al. The irritable hip: immediate ultrasound guided aspiration and prevention of hospital admission. Arch Dis Child 1995;72:110-14.

14 Miralles M, Gonzales G, Pulpeiro J, et al. Sonography of the painful hip in children; 500 consecutive cases. American fournal of Radiology 1989;152:579-82. 